\title{
PREVALENCIA Y PRINCIPALES PATOLOGIAS ASOCIADAS EN NEONATOS CON RETARDO DE CRECIMIENTO INTRAUTERINO DEL HOSPITAL REGIONAL DE ICA, PERU
}

\author{
Prevalence and main pathologies associated in infants with intrauterine growth retardation of the \\ Regional Hospital of Ica, Perú
}

Ismael Rolando Gonzáles Tipiana ${ }^{1, a, d}$ Aracely Guadalupe Rubianes Huamán ${ }^{2, b, c}$

\footnotetext{
${ }^{1}$ Facultad de Medicina, Universidad Nacional San Luis Gonzaga de Ica. Ica, Perú.

${ }^{2}$ Institución Educativa "San Luis Gonzaga" de Ica. Ica, Perú.

${ }^{a}$ Médico Pediatra ${ }^{b}$ Licenciada en Ciencias de la Educación, ${ }^{c}$ Magister en Educación: Administración y Planificación de la Educación Superior, ${ }^{d}$ Doctor en Salud Pública
}

\section{RESUMEN}

Objetivo: Determinar la prevalencia e identificar las principales patologías asociadas en los recién nacidos con retardo de crecimiento intrauterino (RCIU), atendidos en un Hospital de la provincia de Ica, Perú. Material y métodos: Estudio descriptivo, retrospectivo y transversal realizado en el Servicio de Neonatología del Hospital Regional de Ica, durante el periodo comprendido desde el 01 de octubre del 2011 al 30 de septiembre del 2012. Resultados: De 2011 recién nacidos en el periodo de estudio, se determinó que la prevalencia global del retardo de crecimiento intrauterino (RCIU) fue 7,6\% (152 recién nacidos), de los cuales el 71,7\% (109 neonatos) fueron portadores de RCIU asimétrico y el 28,3\% (43 neonatos) de RCIU simétrico. La policitemia fue detectada en el 11,8\% (18 neonatos), la hipoglicemia en el 9,2\% (14 neonatos) y el síndrome de aspiración meconial representó el $3 \%$ (5 neonatos). El 78\%, 119 de los recién nacidos con RCIU tuvieron bajo peso al nacer y el promedio fué 2, 235,8 grs. El 50,7 $\%$ (77 casos) fueron de sexo masculino y 49,3\% (75 casos) de sexo femenino. El 45,4 \%, 69 niños, nacieron por cesárea y vía parto eutócico $54,6 \%, 83$ neonatos. El promedio de Apgar al minuto y a los 5 fué de 8,4 y 8,9 respectivamente. Otras patologías asociadas fueron: trauma obstétrico 4,6\% ( 7 neonatos), malformaciones congénitas 4,6\% (7 neonatos) y asfixia 3,2\% (5 neonatos). La mortalidad fué del 1,31\%. Conclusiones: La prevalencia global de neonatos con retardo de crecimiento intrauterino fue de $7,6 \%$. La morbimortalidad se encuentra incrementada en el grupo de recién nacidos con retardo de crecimiento intrauterino

Palabras claves: Retardo de crecimiento intrauterino, Patologías asociadas.

\section{SUMMARY}

Objective: To determine the prevalence, identify and the main associated pathologies the newborns with intrauterine growth retardation (IUGR) treated at a Hospital in the province of Ica, Peru. Material and Methods: Descriptive, retrospective and cross-sectional study conducted in neonatology of the Ica Regional Hospital service, during the period from October 01, 2011 to September 30, 2012. Results: Of 2011 infants in the study period, it was determined that the overall prevalence of intrauterine growth retardation (IUGR) was 7,6\% (152 newborns), of which $71,7 \%$ (109 newborns) were carriers of asymmetric IUGR and $28,3 \%$ (43 newborns) of symmetric IUGR. Polycythemia was detected in $11,8 \%$ (18 newborns), hypoglycemia in 9,2\% (14 newborns) and meconium aspiration syndrome accounted for $3 \%$ (5 newborns). $78 \%, 119$ of newborns with IUGR had low birth weight and the average was $2,235,8 \mathrm{~g}$. The 50,7\%, 77 newborns were male and 49,3\%, 75 were female. The 45,4\%, 69 children were born via cesarean and vaginal delivery 54,6\%, 83 newborns. The average Apgar minute and 5 was 8,4 and 8,9 respectively. Other associated diseases were: $4,6 \%$ obstetric trauma (7 newborns), congenital malformations 4,6\% (7 newborns) and asphyxia 3,2\% (5 newborns). Mortality was of $1,31 \%$. Conclusions: The global prevalence of infants with intrauterine growth retardation was $7,6 \%$. The morbidity and mortality is increased in the group of infants with intrauterine growth retardation.

Key words: Intrauterine growth restriction, associated pathologies. 


\section{INTRODUCCIÓN.}

El Feto que no alcanza todo su potencial de crecimiento, es un nuevo ser que conlleva implicancias notables en la morbimortalidad perinatal (1). Los avances recientes en la salud fetal con pruebas complementarias y modernas, han permitido disminuir significativamente la mortalidad perinatal, por ello la prevención de la morbilidad del recién nacido, está ocupando cada vez mayor atención en la investigación y asistencia del neonato (2). Los recién nacidos con retardo de crecimiento intrauterino son aquellos niños más pequeños que el promedio, estos neonatos experimentan problemas médicos bien reconocidos que corresponden, a trastornos metabólicos como hipoglicemia, hematológicos como policitemia, síndrome de aspiración de líquido meonial, asfixia, déficit de crecimiento a largo plazo, alteraciones del desarrollo neurológico y altas tasas de mortalidad fetal y neonatal que exceden a las de los recién nacidos normales (3). Recientes evidencias sugieren que adultos que experimentaron retardo de crecimiento intrauterino severo tienen un significativo aumento de patologías tales como; obesidad, hipertensión arterial, resistencia a la insulina, diabetes tipo 2 y alteraciones permanentes a lo largo de la vida en relación al crecimiento y desarrollo $(3,4)$. Estas alteraciones podrían ser consecuencias inevitables de las condiciones ambientales impuestas en la vida intrauterina que conducen a la restricción del crecimiento fetal (4). La observación de esta realidad y la importancia del tema nos llevaron a plantear la presente investigación, cuyo objetivo es determinar la prevalencia del retardo de crecimiento intrauterino y sus principales patologías asociadas en el Hospital Regional de lca entre el $1^{\circ}$ de octubre del 2011 y el 30 de setiembre del 2012.

\section{MATERIAL Y METODOS}

Se realizó un estudio de tipo descriptivo, retrospectivo y transversal para determinar la prevalencia y las principales patologías asociadas en recién nacidos portadores de retardo de crecimiento intrauterino en el Servicio de Neonatología del Hospital Regional de Ica-Perú, durante el período comprendido entre el $1^{\circ}$ de Octubre del 2011 al 30 de setiembre de 2012. Durante este periodo nacieron 2011 niños, de los cuales 152 fueron portadores de retardo de crecimiento intrauterino. Los datos fueron tomados de las historias clínicas maternas y de los recién nacidos y fueron seleccionadas variables como: Edad gestacional (Test de Capurro), sexo, peso, Apgar al nacer y principales causas de morbimortalidad. Se utilizó una ficha de recolección de datos elaborada por el investigador y validada en 30 recién nacidos con RCIU del Hospital Regional de lca $y$ se procesaron estadísticamente usando el programa Epilnfo 2000; asimismo se realizó un análisis univariado y bivariado con IC 95\% considerando un $\mathrm{p}<0,05$ como estadísticamente significativo. Finalmente se confeccionaron tablas, para facilitar el análisis y discusión de los resultados. Dentro de nuestros objetivos se encuentran el determinar la prevalencia de los recién nacidos con RCIU e identificar las principales patologías asociadas: hipoglicemia, policitemia, síndrome de aspiración meconial, asfixia, trauma obstétrico, bajo peso, malformaciones congénitas y mortalidad.

\section{RESULTADOS}

La prevalencia de Retardo de Crecimiento Intrauterino (RCIU) fue 7,6 por cada 100 nacidos vivos, durante el periodo de estudio comprendido entre el 1 de octubre del 2011 al 30 de setiembre del 2012. Se encontró 28,3\%, 43 recién nacidos portadores de RCIU simétrico y $71,7 \%, 109$ con RCIU asimétrico. Los neonatos con RCIU 78,3\%, 119 tenían peso inferior a 2,500 grs, la media alcanzó un valor igual a 2,235.8 grs. con una desviación estándar de 311.5. El 98,7 \%, 150 recién nacidos presentaron una edad gestacional entre 37 a 41 semanas y en el 1,3\%, 2 neonatos se encontró una edad gestacional 
mayor a 41 semanas. La media para la edad gestacional fue 39 semanas y la desviación estándar igual a 1,23. El 45,4\%, 69 nacieron vía parto eutócico y el $54.6 \%, 83$ por cesárea. El promedio de apgar al minuto y a los 5 minutos fue de 8,4 y 8,9 respectivamente. La hipoglicemia se halló con una frecuencia de 9,2\%, 14 recién nacidos y estuvo ausente en $90,8 \%, 138$ recién nacidos con RCIU. La policitemia en los niños con
RCIU fue la patología que se encontró con mayor frecuencia $11,8 \%, 18$ neonatos $y$ estuvo ausente en el 88,2 \%, 134 neonatos. Sólo el 1,9\%, 3 neonatos con RCIU mostraron signos compatibles con síndrome de aspiración meconial, el cual estuvo ausente en el $98,1 \%, 14$ recién nacidos. La mortalidad en los niños portadores de RCIU fue 1,3\%, (2 neonatos).

Tabla № 1: Prevalencia y Principales características de los recién nacidos con retardo de Crecimiento Intrauterino en el Hospital Regional de Ica. 2011-2012

\begin{tabular}{lrrc}
\hline \multicolumn{1}{c}{ VARIABLES } & $\mathbf{n = 1 2 9}$ & $\%$ & $\mathbf{P}$ \\
\hline RCIU & & & \\
Asimétrico & 109 & 72 & 0,05 \\
Simétrico & 43 & 28 & \\
Peso al nacimiento & & & \\
< 2500grs & 119 & 78 & 0,03 \\
> 2500grs & 33 & 22 & \\
Edad gestacional & & & \\
37 - 41 semanas & 150 & 99 & 0,05 \\
$\quad$ 242 semanas & 2 & 1 & \\
Tipo de parto & & & \\
Eutócico & 69 & 45 & 0,41 \\
Cesárea & 83 & 55 & \\
Sexo & & & \\
Masculino & 77 & 51 & 0,04 \\
Femenino & 75 & 49 & \\
Apgar & & & \\
$0-3$ & 1 & 1 & 0,38 \\
$4-6$ & 4 & 3 & \\
$7-10$ & 147 & 96 &
\end{tabular}

TABLAS № 2: Principales patologías asociadas a Retardo de Crecimiento Intrauterino en el Hospital Regional de Ica. 2011-2012

\begin{tabular}{lccc}
\hline VARIABLES & $\mathrm{n}=38$ & $\%$ & $\mathrm{P}$ \\
\hline Policitemia & 18 & 12 & 0,29 \\
Hipoglicemia & 14 & 9 & 0,17 \\
Trauma obstétrico & 7 & 5 & 0,36 \\
M. congénitas & 7 & 5 & 0,43 \\
Asfixia neonatal & 5 & 3 & 0,47 \\
Aspiración meconial & 3 & 2 & 0,54 \\
Mortalidad & 2 & 1 & 0,27
\end{tabular}




\section{DISCUSIÓN}

En esta investigación se determinó que la prevalencia global de retardo de crecimiento intrauterino (RCIU) fue de 7,6\%, 152 neonatos. Asimismo en lo referente al peso de nacimiento se encontró a 119 recién nacidos, con bajo peso inferior a 2,500 grs. La media de peso alcanzó un valor de 2,235,8 grs y una desviación estándar de 311.5. Los hallazgos en relación a la variable peso de los recién nacidos son muy similares a los encontrados por Mere J. y colaboradores, quienes en un estudio realizado en el Hospital Loayza de Lima encontraron, que la media en los recién nacidos con retardo de crecimiento intrauterino alcanzó un valor de 2,389,5 grs. y la desviación estándar fue de 457,7 (5). En relación a la edad gestacional, se encontró en $98,7 \%$ una edad gestacional entre 37 a 41 semanas. La media de la edad gestacional fue de 39 semanas y la desviación estándar alcanzó un valor de 1,23. Al comparar nuestros resultados con los encontrados por otros investigadores observamos que en el Hospital Loayza de Lima, Mere J. y colaboradores, hallaron en los neonatos con retardo de crecimiento intrauterino una media de 38.6 semanas con una desviación estándar de 2,03 (5). Los resultados son muy similares en ambos grupos de estudio. Respecto a la vía de terminación del parto, el $45,4 \%$ nacieron vía parto eutócico y el $54,6 \%$ por cesárea, es decir se observa un ligero predominio de nacimientos por cesárea. En la investigación realizada por Mere J. y colaboradores en el Hospital Loayza de Lima se encontró que el 55,6 \% nacieron vía parto eutócico y el 39,6 \% por cesárea (5). Es decir el porcentaje de nacimientos vía parto eutócico fue superior al de cesáreas. En lo referente al promedio de apgar al minuto y a los 5 minutos este fue de 8,4 y 8,9 respectivamente. Hallazgos similares muestra el estudio realizado en el Hospital Loayza de Lima, donde el promedio de apgar en los niños con retardo de crecimiento intrauterino al minuto y a los 5 minutos fue de 7,6 y $8,7(5)$.
En relación al sexo no se encontró diferencia estadísticamente significativa entre el sexo masculino y femenino. La hipoglicemia estuvo presente en el 9,2\%. La bibliografía revisada menciona una incidencia de hasta de $20 \%$ en los recién nacidos portadores de retardo de crecimiento intrauterino (6). En nuestros resultados, esta patología no alcanza valores tan altos. La incidencia de hipoglicemia en el estudio realizado en el Hospital Nacional Madre Niño "San Bartolomé" de Lima en el año 2,009, fue aún menor a la encontrada en nuestro trabajo de 1,5 a $1,9 \%$ (7).

Referente a la policitemia esta patología estuvo presente en el $11,8 \%$ de los recién nacidos con retardo de crecimiento intrauterino. La bibliografía consultada refiere que la policitemia en el grupo de niños con retardo de crecimiento intrauterino aumenta de 5 a 18\% en comparación con la población general de neonatos (8). Asimismo los investigadores que trabajan en este campo mencionan que la policitemia en ciudades elevadas llega al $5 \%$ y en lugares bajos al 2,7\% (6). El síndrome de aspiración meconial es una patología muy poco frecuente en la población estudiada y de acuerdo a los resultados de nuestra investigación se encontró en el $1,9 \%$ de todos los neonatos con retardo de crecimiento intrauterino. Estos resultados coinciden con las referencias bibliográficas consultadas, las cuales refieren que la incidencia de aspiración meconial es de aproximadamente 1\% (6) En el Hospital Nacional Madre Niño "San Bartolomé" de Lima se encontró una incidencia entre el 0,9 a $2 \%$ de casos (7).

En esta investigación encontramos 28,3\% recién nacidos portadores de retardo de crecimiento intrauterino simétrico y el $71,7 \%$ restante fueron niños con retardo de crecimiento intrauterino asimétrico. Nuestros hallazgos difieren de los encontrados en el estudio realizado por Mere y colaboradores en el Hospital Loayza de Lima, estos autores encontraron un $38,9 \%$ de niños portadores de retardo de crecimiento intrauterino simétrico, 
mientras que el retardo de crecimiento intrauterino asimétrico estuvo presente en el $61,1 \%$ (5). Como puede observarse, hubo cerca de un $10 \%$ más de niños portadores de retardo de crecimiento intrauterino asimétrico. La bibliografía refiere que la mortalidad intraparto y durante la primera semana es muy alta 8 a 10 veces superior que en recién nacidos a término sanos (9). En esta investigación la mortalidad fue 1,3\%.

\section{Correspondencia:}

Dr. Ismael R. Gonzáles Tipiana

Correo electrónico: ismael_w2002@yahoo.es

\section{REFERENCIAS BIBLIOGRAFICAS}

1. Natal A. Prats J. Manual de Neonatología. Madrid: Mosdy / Doyma Libros S.A.; 2010.

2. Gomela TL. Cunningham MD. Eval FG. Zenk KE. Manejo Básico, problemas en la guardia, patologías y farmacoterapia. 3 $3^{\text {a }}$ Ed. Buenos Aires: Médica Panamericana; 2010.

3. Robert C. Vandenbosche, M.D, and Jeffrey T. Kirchner, D.O. Intrauterine Growth Retardation. American Family Physician. 2006. Oct. 15:1-9.
4. Wolman HA. Intrauterine growth restriction: definition and etiology. Horm Res 2009; 49 Suppl 2:1-6

5. Mere J. Jefferson L. Bao V. Izá J. Retardo del Crecimiento Fetal. Ginecología y Obstetricia. 2000 Jul; 46 (3): $1-9$

6. Rodríguez B. R. Manual de Neonatología.1 ${ }^{\text {a }}$ Ed. México D. F: McGraw Hill Interamericana. S. A. 2006.

7. Ministerio de Salud. Hospital Nacional Docente Madre-Niño "San Bartolome". Manual de Procedimientos para el diagnóstico y tratamiento del servicio de neonatología. Lima, Perú. 2009.

8. Avery GB. Tratado de Neonatologia. $6^{\circ}$ Ed. Buenos Aires: Médica Panamericana; 2006

9. Tapia JL. Ventura-Junca T. Manual de Neonatologia. Santiago: Tecnicas Mediterraneo Ltda; 2005

Recibido: 30/08/2014

Aprobado para publicación: 30/11/2014 